\title{
The effect of $n-\mathrm{H}_{2}$ impurity on the heat capacity of solid $\mathrm{Ne}$
}

\author{
M.I. Bagatskii and I.Ya. Minchina \\ B. Verkin Institute for Low Temperature Physics and Engineering of the National Academy of Sciences of Ukraine \\ 47 Lenin Ave., Kharkov 61103, Ukraine \\ E-mail: bagatskii@ilt.kharkov.ua \\ V.S. Parbuzin \\ Lomonosov Moscow State University, Moscow 119899, Russia \\ E-mail: vsp@phys.chem.msu.ru
}

Received October 7, 2008

\begin{abstract}
The alloy of $\mathrm{Ne}-5.1 \% n-\mathrm{H}_{2}$ was investigated calorimetrically under equilibrium vapor pressure in an interval of $0.9-25 \mathrm{~K}$. The magnitudes and the temperatures dependencies of the heat capacity $C_{\mathrm{sol}}(T)$ of the alloy at $T=11-20 \mathrm{~K}$ are strongly dependent on the conditions of sample preparation. The temperatures dependencies of the $C_{\mathrm{sol}}(T)$ of the sample prepared in a calorimeter by direct condensation of a gas mixture at $T \approx 15 \mathrm{~K}$ exhibit a smeared maximum near the triple point temperature of hydrogen and a phase transition at $T=17.1 \mathrm{~K}$. The detected features of $C_{\text {sol }}(T)$ indicate that the preparation of the solid $\mathrm{Ne}-n-\mathrm{H}_{2}$ from a gas phase leads to the formation of a nonequilibrium long-living phase $\mathrm{Ne}$ with large $\mathrm{H}_{2}$ concentrations and a small portion of $\mathrm{H}_{2}$ inclusions with low $\mathrm{Ne}$ concentrations. The phase transition is caused by decompositions of this phase. The phase dose not recover on cooling the sample after the phase transition. It is found that the rate of the ortho-para conversion of the $\mathrm{H}_{2}$ molecules in the solid $\mathrm{Ne}-n-\mathrm{H}_{2}$ solutions higher than that of solid $\mathrm{H}_{2}$.
\end{abstract}

PACS: $65.40 . \mathrm{Ba}$. Heat capacity;

65.60.+a. Thermal properties of amorphous solids and glasses: heat capacity;

65.40.-b. Thermal properties of crystalline solids;

63.50.+x. Vibrational states in disordered systems.

Keywords: heat capacity; hydrogen-containing alloys; phase transitions.

\section{Introduction}

The solid $\mathrm{Ne}-\mathrm{H}_{2}\left(\mathrm{Ne}-\mathrm{D}_{2}\right)$ mixtures prepared by condensing a gas phase directly into a solid one can form nonequilibrium long-living semiquantum objects - new phases of solutions, molecular complexes $\mathrm{Ne}\left(\mathrm{H}_{2}\right)_{n}$ $\left(\mathrm{Ne}\left(\mathrm{D}_{2}\right)_{n}\right)$ and clusters $\mathrm{Ne}\left(o-\mathrm{H}_{2}\right),\left(\mathrm{Ne}\left(p-\mathrm{D}_{2}\right)\right)$ [1-7]. The formation of these objects leads to new features of the physical properties of the system. Investigations of solid $\mathrm{Ne}-\mathrm{H}_{2}$ solutions by different physical method are also important for solving technological problems of storage of high energy-capacitive states.

Solid $\mathrm{Ne}-\mathrm{H}_{2}$ solutions were investigations thoroughly by $\mathrm{x}$-ray methods in a wide concentration range from 0.5 to $98 \mathrm{~mol} \% \mathrm{H}_{2}$ obtained by depositing gas mixture on a substrate at $T \sim 5 \mathrm{~K}[1-3]$. It is found [1,2] that the vacuum $\mathrm{Ne}-\mathrm{H}_{2}$ condensates contain a metastable long-living hexagonal hcp 2 phase of $\mathrm{Ne}$ together with phases based on the hcp $\mathrm{p}_{1}$ and fcc lattice of the initial components. The volume unit-sell of the hcp 2 phase is close to that of the pure Ne lattice. The quantity of the $\mathrm{hcp}_{2}$ phase increases with the $\mathrm{H}_{2}$ concentration in the starting mixtures and the fcc phase decreases linearly. The $\mathrm{hcp}_{2}$ phase can contain up to $83 \% \mathrm{H}_{2}$ in the limiting case [1]. On approaching the melting temperature of the crystals, the $\mathrm{hcp}_{2}$ phase transforms irreversibly into the phases that were present in the starting components occurs near the melting point of the crystals. The authors [1] attribute the formation of the $\mathrm{hcp}_{2}$ in the condensates to strongly nonequilibrium conditions of crystallization of the gas mixtures at $T \sim 5 \mathrm{~K}$.

The goal of this study was use calorimetric methods for investigating solid $\mathrm{Ne}-5.1 \mathrm{~mol} . \% n-\mathrm{H}_{2}$ solutions samples prepared in a calorimeter by direct condensation of a gas mixture and crystallization of a liquid in the interval $0.9-25 \mathrm{~K}$. 


\section{Results and discussion}

The experiment was performed using an adiabatic calorimeter [8]. The calorimetric heating lasted 2-6 min. The time taken to achieve a nearly steady-state temperature condition in the calorimeter since the onset of heating varied within 10-40 min. The purity of the starting components was no worse than $99.99 \%$ (here and below we refer to molar fractions). The sample mass $(\sim 0.5 \mathrm{~mol})$ and the $\mathrm{H}_{2}$ concentration in the mixture were estimated by the $P-V-T$ method the error being $0.2 \%$ and $0.5 \%$, respectively. The measurements error in the heat capacity of the solution was $4 \%$ at $0.9 \mathrm{~K}, 1 \%$ at $2 \mathrm{~K}$ and about $0.5 \%$ above $4 \mathrm{~K}$.

The differences in the heat capacity under equilibrium vapor tension and constant pressure are negligible in the investigated interval of temperature.

A solid sample was first condensed in a calorimeter directly from a gas mixture at $T \approx 15 \mathrm{~K}$. Four successive series of measurement of heat capacity $C_{\mathrm{sol}}(T)$ were performed on the starting solid sample: $1-\delta T=5-7.5 \mathrm{~K}, 2-\delta T=4-16$ $\mathrm{K}, 3-\delta T=11-21 \mathrm{~K}, 4-\delta T=12.5-18 \mathrm{~K}$. After series 4 the time dependence of the sample temperature (thermogram) was measured in the interval $22-24.7 \mathrm{~K}$ and the sample was melted. The liquid sample was held for 10 hours at $T=25 \mathrm{~K}$ (the temperature of the triple point of $\mathrm{Ne}$ is $24.56 \mathrm{~K})$ then crystallized, cooled to $0.85 \mathrm{~K}$ and measurement series $5(\delta T=0.9-18 \mathrm{~K})$ and $6(\delta T=12.9-15.4 \mathrm{~K})$ were performed.

It is seen that the results of series 1-3 coincide (see Fig. 1). The dependences $C_{\text {sol }}(T)$ have some peculiarities: (i) there is a small smeared maximum at the triple point temperature of hydrogen (for $n-\mathrm{H}_{2} 13.93 \mathrm{~K}$ ); (ii) a phase

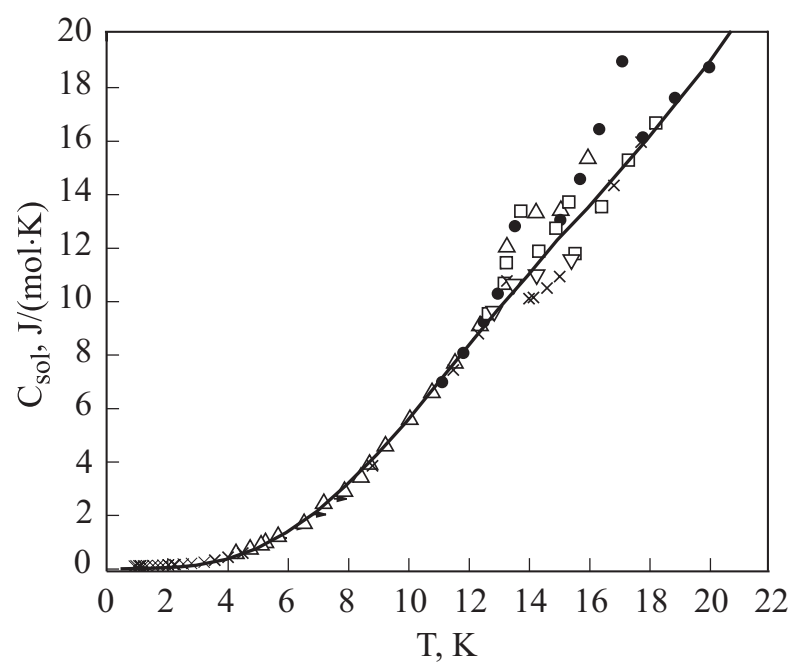

Fig. 1. Heat capacity $C_{\text {sol }}(T)$ of solid $\mathrm{Ne}-5.1 \mathrm{~mol} \% \mathrm{n}-\mathrm{H}_{2}$ solution: Series $1(+), 2(\Delta), 3(\bullet), 4(\square)$ are successive measurements of the heat capacity of the solid sample prepared from a gas phase; Series $5(X)$ and $6(\nabla)$ are heat capacity measured on the solid sample obtained from a liquid phase. The carve shows smoothed experimental values of heat capacity of solid $\mathrm{Ne}$ [9]. transition is detected near $T=17.1 \mathrm{~K}$. The results of series $1-3$ and $4-6$ coincide at $T<12 \mathrm{~K}$. In the interval $13-17 \mathrm{~K}$ the heat capacities measured in series $4-6$ are dependent on the temperature prehistory of the sample. The features detected in $C_{\text {sol }}(T)$ can be interpreted as follows. On condensation from a gas phase (near $T=15 \mathrm{~K}$ ) the solid $\mathrm{Ne}-5.1 \% n-\mathrm{H}_{2}$ solution can develop a nonequilibrium long-living phase $\mathrm{Ne}$ with large $\mathrm{H}_{2}$ concentrations in addition to the fcc phase of Ne. This phase has hcp lattice [1]. The phase transition destroys the hcp phase which does not recover on the subsequent cooling the sample. The anomalous behavior of $C_{\text {sol }}(T)$ in the region $13-15 \mathrm{~K}$ is caused by the inclusions of $\mathrm{H}_{2}$ with low-concentration $\mathrm{Ne}$ present in the solution.

At $T=13-16 K C_{\text {sol }}(T)$ exhibits quite different behavior in solid solutions prepared by crystallization from the liquid phase and by condensation of the gas phase. This points to significant structural distinctions in samples obtained by different techniques. The gas condensation produces a solid sample with on the average homogeneous composition of the components. The solid sample crystallized from the liquid phase an inhomogeneous composition of the components. Solid $\mathrm{Ne}$ with a low $\mathrm{H}_{2}$ concentration makes most of the sample. The upper part of the sample contains $\mathrm{H}_{2}$ with a low Ne concentration. There are gradients of the component concentrations along the height of the sample. The behavior of the dependence $C_{\text {sol }}(T)$ in measurement series 5 can be explained assuming that the $\mathrm{H}_{2}$ phase is amorphous, nonequilibrium and frozen. The assumption is supported by the behavior of $C_{\text {sol }}(T)$ in measurement series 6 .

The experimental results of the heat capacity of solid Ne-5.1 mol.\% $n-\mathrm{H}_{2}$ solution measured at $T<3 \mathrm{~K}$ (series 5)

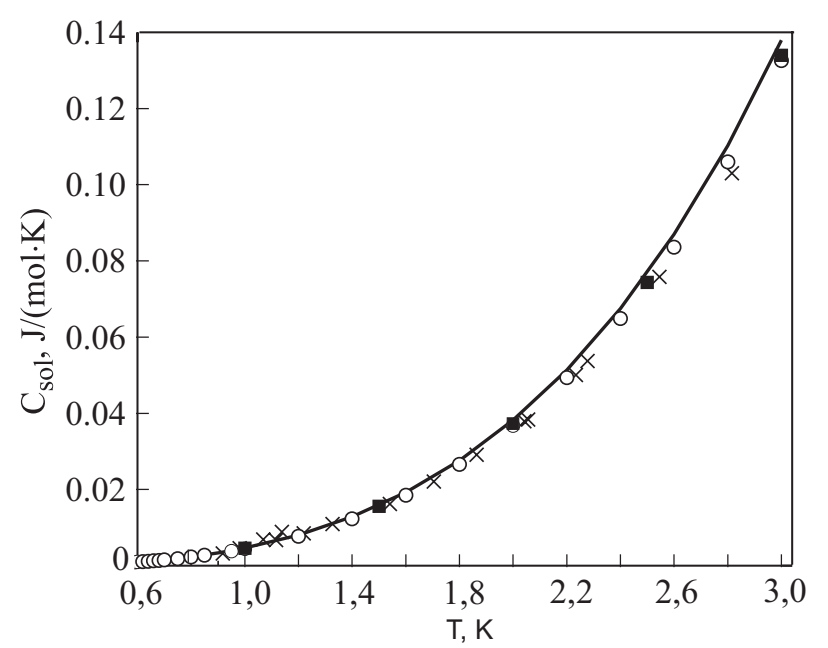

Fig. 2. Heat capacity $C_{\text {sol }}(T)$ of solid $\mathrm{Ne}-5.1 \mathrm{~mol} \% \mathrm{n}^{-\mathrm{H}_{2}}$ solution in the low temperature region: $\times$ - our experimental results (measurement series 5); ( $\mathbf{\square}$ - smoothed experimental values of heat capacity of solid $\mathrm{Ne}$ [9]; o - a sum of the heat capacity of $p-\mathrm{H}_{2}(5.1 \%)$ and $\mathrm{Ne}(94.9 \%)$. The curve presents the heat capacity of solid Ne calculated by Eq. (1). 
are shown in Fig. 2. It is seen that our results, the heat capacity of solid $\mathrm{Ne}$ [9] and the sum of $p-\mathrm{H}_{2}(5.1 \%)$ and $\mathrm{Ne}$ $(94.9 \%)$ differ less than by $7 \%$.

It is shown theoretically [10] that the lattice specific heat of solids with central short-range forces of interaction and close-packed lattices can be described at $T \sim 1 \mathrm{~K}$ by the following expression:

$$
C_{V}=2.4 \pi^{4} R\left(T / \theta_{0}\right)^{3}\left[1+\alpha_{1}\left(T / \theta_{0}\right)^{2}+\alpha_{2}\left(T / \theta_{0}\right)^{4}\right],
$$

where $\theta_{0}$ is the limiting Debye temperature at $T \rightarrow 0 \mathrm{~K}$; $\alpha_{1}$ and $\alpha_{2}$ are constants. It is found $[8,11]$ that the heat capacity of quantum and classical crystals ${ }^{4} \mathrm{He}, p-\mathrm{H}_{2}, o-\mathrm{D}_{2}$, $\mathrm{HD}, \mathrm{Ne}, \mathrm{Ar}, \mathrm{Kr}$ and $\mathrm{Xe}$ can be described at $T / \theta_{0} \leq 0.04$ quite adequately by Eq. (1) (law of corresponding states) using the averages $\alpha_{1}=47$ and $\alpha_{2}=1.5 \cdot 10^{-4}$. The law of corresponding states obeyed for the heat capacity of solid $\mathrm{Ne}$ at $\theta_{0}=75.1 \mathrm{~K}$ [9] is shown in Fig. 2 .

It is seen that the contribution to the heat capacity of the solution from the rotational subsystem of the $o-\mathrm{H}_{2}$ molecules with the rotational quantum number $J=1$ is negligible. Measurement series 5 was started 200 hours after preparation of the solid sample.

The rate of the change in the $o-\mathrm{H}_{2}$ concentration in solid hydrogen due to the ortho-para conversion is described as [12]

$$
d x / d t=-k x^{2},
$$

with the general solution

$$
1 / x=1 / x_{0}+k t,
$$

where $k=(1.90 \pm 0.03) \cdot 10^{-2} \mathrm{~h}^{-1}[13]$ is the conversion constant, $x_{0}(t=0)=75 \%$ is the starting $o-\mathrm{H}_{2}$ concentration at $t=0 \mathrm{~h} . t=0 \mathrm{~h}$ was taken to denote the moment of the end of the substance condensation to the calorimeter $T \sim 15 \mathrm{~K}$. According to calculation by Eg. (2), the concentration of the ortho-para molecules in solid hydrogen decreases during $200 \mathrm{~h}$ from $75 \%$ to $19 \%$. According to $[8,14,15]$ the rotational subsystem of $19 \%$ of the $o-\mathrm{H}_{2}$ molecules makes a significant contribution to the heat capacity of the solution. The discrepancy can be explained assuming that the rate of the ortho-para conversion hydrogen molecules in a solid solution is much higher than it is in solid $\mathrm{H}_{2}$ under equilibrium vapor pressure. The molar volumes of $\mathrm{H}_{2}\left(23.06 \mathrm{~cm}^{3} / \mathrm{mol}\right)$ and $\mathrm{Ne}\left(13.31 \mathrm{~cm}^{3} / \mathrm{mol}\right)$ differ significantly [16]. The molar volumes of the nonequilibrium long-living phase $\mathrm{Ne}$ in the solid solution is close to that of solid $\mathrm{Ne}[1,2]$. The measurements at higher pressures [17-19] show that the conversion rate increases sharply with the density of solid $\mathrm{H}_{2}$.

We measured the dependence of the resistance thermometer $R(t)$ in the interval $21-24.7 \mathrm{~K}$ (thermogram). A part of the thermogram measured in the region of the sample melting is shown in Fig. 3. $R(t)$ exhibits no anomalous features. The temperature smearing of the sample melting is about

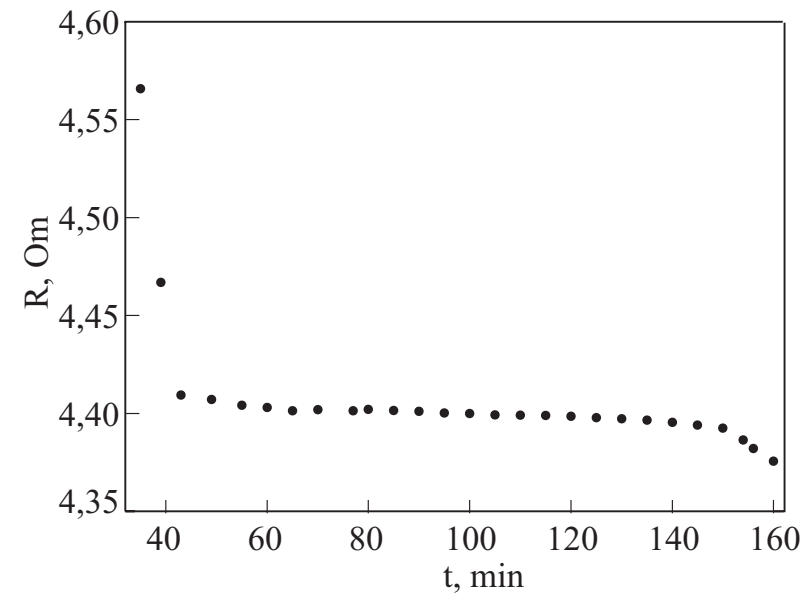

Fig. 3. Time dependence of the resistance thermometer $R(t)$ (thermogram) in the region of solution melting.

$0.1 \mathrm{~K}$. The melting of the sample ended at $T=24.54 \mathrm{~K}$. The triple point temperature of $\mathrm{Ne}$ is $24.56 \mathrm{~K}$ [16].

\section{Conclutions}

Two sample of solid $\mathrm{Ne}-5.1 \% n-\mathrm{H}_{2}$ were investigated calorimetrically in the interval of $0.9-25 \mathrm{~K}$. Sample 1 was prepared in a calorimeter at $T \approx 15 \mathrm{~K}$ by condensing a gas mixture directly to a solid phase. Sample 2 was obtained in a calorimeter by crystallizing a liquid (after melting and holding the liquid solution for 10 hours at $T=25 \mathrm{~K})$.

The main qualitative results of this study are as follows.

1. It has been found experimentally that the magnitudes and the temperatures dependencies of the heat capacity $C_{\text {sol }}(T)$ of the solid samples differ significantly in the region $11-20 \mathrm{~K}$. The dependences $C_{\mathrm{sol}}(T)$ have some peculiarities: (i) there is a small smeared maximum at the triple point temperature of hydrogen $(13.93 \mathrm{~K})$; (ii) a phase transition is detected near $T=17.1 \mathrm{~K}$. The features detected in $C_{\text {sol }}(T)$ can be interpreted as follows. On condensation from a gas phase (near $T=15 \mathrm{~K}$ ) the solid $\mathrm{Ne}-$ $5.1 \% n-\mathrm{H}_{2}$ solution can develop a nonequilibrium long-living phase $\mathrm{Ne}$ (with large $\mathrm{H}_{2}$ concentrations) in addition to the fcc phase of Ne. This phase has hep lattice [1]. The phase transition destroys the hcp phase which does not recover on the subsequent cooling the sample. The anomalous behavior of $C_{\text {sol }}(T)$ in the region $13-15 \mathrm{~K}$ is caused by the inclusions of $\mathrm{H}_{2}$ with low-concentration Ne present in the solution.

2 . The rate of the ortho-para conversion of the $\mathrm{H}_{2}$ molecules in the solid $\mathrm{Ne}-5.1 \% n-\mathrm{H}_{2}$ solutions is higher than that of solid $\mathrm{H}_{2}$. The effect is due to the higher density of the solution in comparison to the density of $\mathrm{H}_{2}$. According to [17-20], the conversion rate increases sharply with the density of solid hydrogen. 
The authors are indebted to A.I. Prokhvatilov, and M.A. Strzhemechny for helpful discussions.

1. N.N. Galtsov, A.I. Prokhvatilov, and M.A. Strzhemechny, Fiz. Niz. Temp. 30, 1307 (2004) [Low Temp. Phys. 30, 984 (2004)].

2. A.S. Barylnik, A.I. Prokhvatilov, and G.N. Shcherbakov, Fiz. Niz. Temp. 21, 787 (1995) [Low Temp. Phys. 21, 607 (1995)].

3. A.S. Barylnik, A.I. Prokhvatilov, M.A. Strzhemechny, and G.N. Shcherbakov, Fiz. Niz. Temp. 19, 625 (1993) [Low Temp. Phys. 19, 447 (1993)].

4. M.I. Bagatskii, I.Ya. Minchina, and V.G. Manzhelii, Fiz. Niz. Temp. 22, 53 (1996) [Low Temp. Phys. 22, 37 (1996)].

5. V.G. Manzhelii, V.A. Popov, G.P. Chausov, and L.I. Vladimirova, J. Low Temp. Phys. 14, 397 (1974).

6. V.B. Kokshenev, Fiz. Nizk. Temp. 2, 236, (1976) [Sov. J. Low. Temp. Phys. 2, 118, (1976)].

7. M.I. Bagatskii, I.Ya. Minchina, and V.M. Bagatskii, Fiz. Niz. Temp. 31, 620 (2005) [Low Temp. Phys. 31, 470 (2005)].

8. M.I. Bagatskii, I.Ya. Minchina, and V.G. Manzhelii, Fiz. Niz. Temp. 10, 1039 (1984) [Sov. J. Low Temp. Phys. 10, 542 (1984)].

9. R.Q. Fugate and C.A. Swenson, J. Low Temp. Phys. 10, 317 (1973).
10. T.H. Barron and J.A. Morrison, Canad. J. Phys. N35, 799 (1957).

11. M.I. Bagatskii, I.Ya. Minchina, V.G. Manzhelii, P.I. Muromtsev, A.I. Krivchikov, and V.C. Parbuzin, Fiz. Niz. Temp. 16, 1009 (1990) [Sov. J. Low Temp. Phys. 16, 589 (1990)].

12. K. Motizuki and T. Nagamiya, J. Phys. Soc. Jpn. 11, 93 (1956).

13. F. Schmidt, Phys. Rev. B10, 4480 (1974).

14. I.Ya. Minchina, M.I. Bagatskii, V.G. Manzhelii, and A.I. Krivchikov, Fiz. Niz. Temp. 10, 1051 (1984) [Sov. J. Low Temp. Phys. 10, 549 (1984)].

15. R.W. Hill, Philos. Mag. 45, 277 (1954).

16. V.G. Manzhelii, A.I. Prokhvatilov, I.Ya. Minchina, and L.D. Yantsevich, Handbook of Binary Solutions of Cryocrystals, Begell House, inc., New York, Wallingford, UK (1996).

17. R.F. Buzerak, M. Chan, and H. Meyer, J. Low Temp. Phys. 28, 415 (1977).

18. G. Ahlers, J. Chem. Phys. 40, 3123 (1964).

19. P. Pedroni, H. Meyer, F. Weinhaus, and D. Haase, Solid State Commun. 14, 279 (1974).

20. M.A. Strzhemechny and R.J. Hemley, Phys. Rev. Lett. 85, 5595 (2000). 